$\%$ ) cases. More patients with rheumatoid-factor negative (54 vs $50 \%$; $p=0.04$ ) and rheumatoid-factor positive pJIA (13 vs 10\%, $p=0.04$ ) received combination with MTX, while more patients in the monotherapy cohort had extended oligoarthritis (40 vs $32 \%, \mathrm{p}<0.001$ ). Patients with MTX had a shorter disease duration ( 4 vs 5.5years, $\mathrm{p}<0.001$ ) and received concomitant steroid more often (34 vs $24 \%$ ), $p<0.001)$. There was no statistical difference regarding disease activity parameters (active joint count, patient assessment and physician assessment of disease activity, ESR, CRP, CHAQ-DI, JADAS10). Discontinuation due to ineffectiveness was reported for ETA/ADA/GOL/TOC in $20 \% / 18 \% / 14 \% / 28 \%$ of patients, respectively in 3.7/4.9/6/10.5 patients/100 treatment years. Thus discontinuation due to inefficacy was reported less frequently with ETA compared to ADA $(p=0.046)$ and TOC $(p<0.001)$ and with ADA compared to TOC $(\mathrm{p}<0.001)$.

Patients on ETA and ADA had a slightly, but not statistically significant lower rate of withdrawal for ineffectiveness if on methotrexate (Figure 1). There was no difference regarding baseline disease activity parameters in patients with ETA/ADA monotherapy compared with combination with MTX, apart from patients with ETA+MTX receiving more often systemic steroids at baseline (36vs $24 \%$, p $<0$ 001). For both GOL and TOC treatment, no baseline differences in disease activity between cohorts with monotherapy and MTX combination could be shown. The combination with MTX led to significantly lower rates of discontinuation due to inefficacy $(p<0.05)$ with GOL and TOC (Figure 1).

Conclusion: Patients with pJIA mostly were treated with a combination of the biologic and MTX rather than with biologic monotherapy. Treatment was discontinued due to lack of efficacy in $14 \%$ to $28 \%$. No statistically significant effect of combination treatment with MTX versus monotherapy could be observed regarding the rate of treatment failures in patients treated with ETA or ADA. However, combination treatment with MTX significantly prolonged the survival of GOL and TOC in patients with polyarticular JIA. The results are limited by low patient numbers in the GOL cohort and possible bias by JIA category.
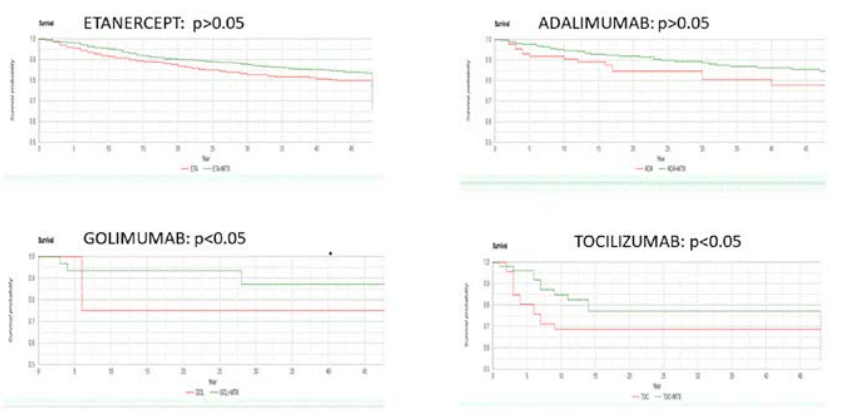

Figure 1. Kaplan Meier plot of drug survival in patients with monotherapy or with combination with MTX of the indicated biologic

Disclosure of Interests: Gerd Horneff Speakers bureau: MSD, Grant/research support from: Pfizer, Roche, Chugai, MSD, Daniel Windschall: None declared, Kirsten Minden Speakers bureau: Pfizer, Abbvie, Grant/research support from: Pfizer, Toni Hospach: None declared, Frank Dressler: None declared, Frank Weller-Heinemann: None declared, Boris Huegle: None declared, Ivan Foeldvari Speakers bureau: Pfizer, Ariane Klein: None declared DOI: 10.1136/annrheumdis-2021-eular.995

\section{POS1302 THE MUSCULOSKELETAL SYSTEM MANIFESTATIONS IN CHILDREN WITH FAMILIAL MEDITERRANEAN FEVER}

F. Demir ${ }^{1}$, S. Canbek ${ }^{2}$, B. Sözeri ${ }^{1} .{ }^{1}$ Health Sciences University, Umraniye Training and Research Hospital, Pediatric Rheumatology, Istanbul, Turkey; ${ }^{2}$ Health Sciences University, Umraniye Training and Research Hospital, Medical Genetics, Istanbul, Turkey

Background: Familial Mediterranean fever (FMF) is a monogenic inherited periodic fever syndrome presenting with episodes of self-limiting fever and inflammation of serosal membranes. The attacks emerge with arthritis were defined as one of the major diagnostic criteria besides involvement of serosal membranes. Non-specific musculoskeletal findings such as myalgia, arthralgia, transient synovitis, and more rare manifestations like protracted febrile myalgia can also be seen in FMF patients attacks

Objectives: We aim to reveal the frequency and genotype association of musculoskeletal manifestations in children with FMF.

Methods: The patients diagnosed with FMF between January 1, 2017 and June 1,2019 , and followed for at least 6 months in our pediatric rheumatology clinic were included in the study. Musculoskeletal manifestations of patients were enrolled. The patients were grouped according to the "Mediterranean Fever" (MEFV) gene variants. Musculoskeletal manifestations of the patients were compared between the groups

Results: The study group included 634 children with FMF (336 female and 298 male, F/M: 1.13/1). The clinical manifestations of patients in attack period were as follows: $99 \%$ of the patients had fever, $87.3 \%$ had abdominal pain, $20.7 \%$ had chest pain, $11.3 \%$ had vomiting, $10.7 \%$ had erysipelas like erythema, and $9.3 \%$ had headache. The musculoskeletal symptoms were accompanied by $58.6 \%$ ( $\mathrm{n}: 372$ ) of the patients during the attack period. The most common musculoskeletal manifestation was found as arthralgia $(32.6 \%$, $\mathrm{n}:$ 206). Also, the other musculosceletal manifestations were found as follows during attacks; arthritis in $23.7 \%$ ( $n: 150)$, myalgia in $20.5 \%$ ( $n: 130$ ) exertional calf pain in 6.5\% (n: 41$)$, and protracted febrile myalgia in $1 \%(n$ 7) of the patients. It was observed that the musculoskeletal manifestations were significantly higher in patients with homozygous M694V variant in exon$10(p=0.017)$. Also, it was found that the musculoskeletal manifestations are more common in the attack periods of patients carrying the M694V variant in at least one allele $(p=0.019)$.

Conclusion: It was determined that the musculoskeletal manifestations were seen as an attack symptom in more than half of FMF patients. Also, homozygous and compound heterozygous MEFV mutations including M694V variant found as a risk factor for emerge of musculoskeletal manifestations. In children with unexplained and recurrent musculoskeletal symptoms, especially in ethnicities with the high frequency of FMF, analysis of MEFV gene can help reveal the underlying cause.

\section{REFERENCES:}

[1] Brik R, Shinawi M, Kasinetz L, Gershoni-Baruch R. The musculoskeletal manifestations of familial Mediterranean fever in children genetically diagnosed with the disease. Arthritis Rheum 2001;44:1416-9.

[2] Jarjour RA, Dodaki R. Arthritis patterns in familial Mediterranean fever patients and association with M694V mutation. Mol Biol Rep 2011;38:2033-6.

Disclosure of Interests: None declared

DOI: 10.1136/annrheumdis-2021-eular.1169

\section{POS1303 \\ EXPERIENCE WITH ADALIMUMAB BIOSIMILAR USE IN CLINICAL PRACTICE: DATA FROM THE GERMAN BIKER-REGISTRY}

G. Horneff ${ }^{1}$, F. Dressler ${ }^{2}$, M. Rühlmann ${ }^{3}$, T. Geikowski ${ }^{4}$, S. Mrusek ${ }^{5}$, A. Klein ${ }^{1}$.

${ }^{1}$ Asklepios Clinic Sankt Augustin, General Paediatrics, Sankt Augustin,

Germany; ${ }^{2} \mathrm{MHH}$, Paediatrics, Hannover, Germany; ${ }^{3}$ Office, Paedaitrics,

Goettingen, Germany; ${ }^{4}$ Office, Paediatrics, Bad Honnef, Germany; ${ }^{5}$ Office,

Paediatrics, Baden-Baden, Germany

Background: In 2017, Adalimumab Biosimilars became approved. Comparative studies to the originator have been performed in adult patients with rheumatoid arthritis, ankylosing spondylitis and psoriasis and extrapolation led to approval for juvenile idiopathic arthritis (JIA).

Objectives: So far there is limited experience with biosimilars in JIA: The large national data base of the BIKER-registry was used to describe experience with Adalimumab biosimilars in clinical practice

Methods: This retrospective analysis used data of the German BIKER-registry. The data basis war screened for patients exposed to Adalimumab. Subcohorts with initiation of treatment after 2017, use of the originator and of biosimilars were built. The course of JADAS10, Physician global assessment VAS 0-100$\mathrm{mm}$, Parent/patient global assessment VAS 0-100-cm, Active joint count 0-71, truncated at 10, ESR and CHAQ-DI was analyzed. Descriptive statistics was used for demographic, clinical data, drug exposure, adverse events (AEs) and events of special interest (ESI).

Results: Until 31.10.2020, 1173 JIA patients were reported to have received Adalimumab. 352 treatments have been started after January 1, 2017. A biosimilar was used first line in 44 patients. Further 55 patients switched for the originator to a biosimilar. 2 patient switched from a biosimilar to the originator. 3 patients switched to a second biosimilar while 5 patients who switched from the originator to a biosimilar reswitched back to the originator.

After 2017, 33 pediatric rheumatology centres reported initiation of Adalimumab treatment. 17 have used a biosimilar. 15 centres have swichted at least 1 patient from the originator to a biosimilar and 14 have used first line a biosimilar in at least 1 patient. In a single centre, initiation of a biosimilar was used more frequently (8 versus 7 ).

The patients' characteristics and disease activity parameters were brightly comparable. The JIA category rheumatoid factor (RF) negative polyarthritis was less frequent in the biosimilar first cohort while RF positive polyarthritis and psoriatic arthritis was more frequent. In patients with idiopathic uveitis the originator was used more often. In the switching cohort, more patients had RF negative polyarthritis, persistent oligoarthritis but less had psoriatic arthritis and no had RF positive polyarthritis. 